\title{
Spin-dependent diastereoselectivity in the photocycloaddition of aldehydes to 2,2-dimethyl-2,3-dihydrofuran
}

\author{
Samir Bondock ${ }^{1,2}$ and Axel G. Griesbeck ${ }^{2, \dagger}$ \\ ${ }^{1}$ Department of Chemistry, University of Mansoura, Egypt \\ ${ }^{2}$ Institute of Organic Chemistry, University of Cologne, Greinstr. 4, D-50939 Köln, Germany
}

\begin{abstract}
The simple (non-induced) diastereoselectivity of the photocycloaddition of aliphatic as well as aromatic aldehydes to 2,2-dimethyl-2,3-dihydrofuran (1) was analyzed as a function of the substrate concentration (spin mapping) and rationalized in terms of optimal spin-orbit coupling controlled triplet biradical geometries.
\end{abstract}

\section{INTRODUCTION}

The technique of "spin-mapping" of diastereoselectivities of bimolecular photochemical reactions is of special relevance in reactions of electronically excited molecules and decisive in controlling and manipulating the stereochemistry of photocycloaddition reactions [1]. In a series of publications, we have recently described the effect of substrate concentration on the spin-selectivity [2] of the Paternò-Büchi reactions of aliphatic aldehydes with 2,3-dihydrofuran [3], allylic alcohols [4], and cyclooctene [5], as well as temperature and viscosity dependence of these reactions [6]. More recently, we have also investigated the ${ }^{2} \mathrm{H}$ magnetic isotope effect on the stereoselectivity in singlet and triplet photocycloaddition reactions [7]. Paternò-Büchi reactions with singlet excited carbonyls most probably proceed via conical intersections, the reactions between triplet excited carbonyls and alkenes pass through triplet 1,4-biradical intermediates [8]. Whereas benzaldehyde and derivatives are applied as reactive triplet species, naphthaldehydes can be used in Paternò-Büchi reactions as reactive singlet excited components [9]. The Paternò-Büchi reaction of $\beta$-naphthaldehyde with 2,3-dihydrofuran is a singlet process (as shown by-non productive-triplet sensitization experiments) and gave predominantely the thermodynamically favoured exo-diastereoisomer. Griesbeck and Mattay have reported that an increase in steric demand of the $\alpha$-substituent at the carbonyl component improved the endo/exo ratio. Methyl trimethylpyruvate with the sterically more demanding tert.-butyl group gave solely the thermodynamically disfavoured endo-diastereoisomeric oxetane (d.s. $>98 \%$ ) with a dioxolene substrate [10]. Similar results were obtained for 2,2-bis-isopropyl-1,3-dioxolene; in this case the endo/exo-ratio of the bicyclic oxetane

${ }^{\dagger}$ E-mail: griesbeck@uni-koeln.de product is inverted when going from the triplet excited mesitylaldehyde to the singlet excited naphthaldehyde as substrate. Two structural features made the conformational analysis of spin-inversion geometries straightforward: the two sites of the alkene part were strongly differentiated concerning the degree of substitution and steric hindrance. The alkenes investigated in these studies were always cyclic, thus reducing the conformational flexibility at the biradical stage. The situation became more complex when one or both of these structure features is modified. Methyl-substituted cycloalkenes have two ISC-reactive sites and thus, the endo/exo-ratios dropped significantly [11]. An extreme example was 1,2-dimethylcyclobutene: the Paternò-Büchi reaction of this substrate with benzaldehyde resulted solely in the exo-diastereomer. This is exactly predicted by the "ISC-geometry model" [12] which is depicted in Figure 1 and relates to an orthogonal approach of the radical centers in a triplet 1,4-biradical because of an optimized spin-orbit coupling (SOC) value [13].

In case of the bis-methylated cycloalkene, one side is now sterically more demanding (compared to unsubstituted starting materials) and the biradical combination trajectory involves the approach from the less shielded cyclobutene plane. Steric hindrance can also reach a critical value during bond formation and might favor the formation of the thermodynamically more stable product. Park and coworkers [14] described the photocycloaddition of benzaldehyde to 2,2-diethoxy-3,4-dihydro-2H-pyran which gave preferentially the exo-phenyl product (d.e. 92\%).

In order to expand our recent studies on the spin-mapping of endo/exo-diastereoselectivities, we envisaged to modify the cycloalkene part in such a way that the thermodynamic preference of the exodiastereoisomer is strongly enhanced. From such an approach we expected a more distinct effect on the 


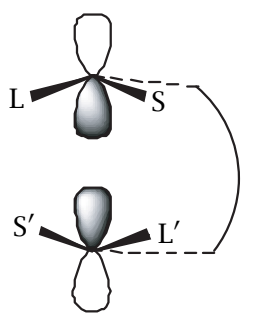

"singlet approach"

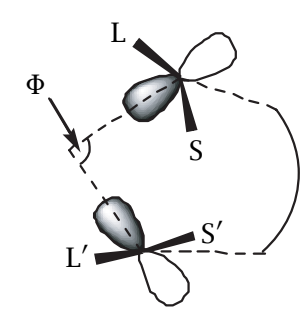

“triplet approach"
Figure 1. The "ISC-geometry model": optimal spin-orbit coupling geometries in triplet $1, n$-biradicals.

spin-orbit coupling controlled diastereoselectivity of the triplet photocycloaddition. In order to "block" the ring side of the cycloalkene, we introduced two methyl groups proximate to the oxygen atom of the 2,3dihydrofuran substrate, i.e., at the C2-position.

\section{RESULTS AND DISCUSSION}

In this study, 2,2-dimethyl-2,3-dihydrofuran (1) was applied as a "ring-side blocked" substrate. This cycloalkene was prepared by a three step reaction according to a literature procedure [15]. In order to estimate if there is a correlation between spin selectivity and steric effects concerning the simple diastereoselectivity of the Paternò-Büchi reaction, the photocycloaddition of 1 with aliphatic and aromatic aldehydes was studied. As typical triplet carbonyl precursors, benzaldehyde (2a) and o-toylaldehyde (2b) were used. Additionally, four aliphatic aldehydes (2c-f) which can react either from their singlet as well as triplet excited states were irradiation with $\mathbf{1}$ in equimolar ratios in benzene (Scheme 1).

Both benzaldehyde and o-tolualdehyde, having high intersystem crossing (ISC) rates and thus exclusively reacting via their first excited triplet states with 2,2dimethyl-2,3-dihydrofuran (1), showed high endo/exo selectivities (see Table 1, entries A and B, respectively). The selectivities were nearly identical to those with the unsubstituted 2,3-dihydrofuran as alkene component (88: 12 and $90: 10$, respectively [16]).

From recent concentration studies [3], we experienced that aliphatic aldehydes can equally react from both singlet and triplet excited states at specific concentrations (termed "isospin selectivity"). The photocycloaddition of the electronically excited propionaldehyde (2c) at high concentrations (1.0 M, singlet-like conditions) to 2,2-dimethyl-2,3-dihydrofuran (1) resulted in the oxetane 3c with a moderate endo/exo selectivity (d.r.: 62 : 38) whereas the reaction at low concentrations of propionaldehyde ( $0.1 \mathrm{M}$, triplet-like conditions) showed an increase in endo-selectivity (d.r.: $74: 26$ ) (see

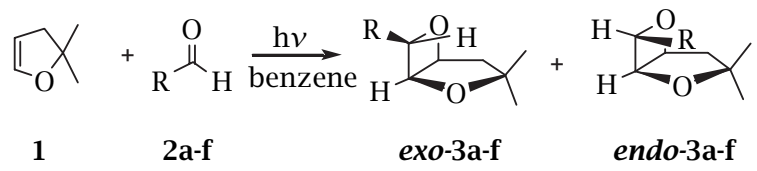

Scheme 1. Paternò-Büchi reaction with 2,2-dimethyl-2,3dihydrofuran (1).

Table 1. Results of photolysis of 2,2-dimethyl-2,3dihydrofuran with aldehydes.

\begin{tabular}{cccc}
\hline entry & $\mathrm{R}=$ & Conc. $(\mathrm{M})$ & endo/exo-3a-f \\
\hline A & Ph & 0.1 & $86: 14$ \\
B & o-Tol. & 0.1 & $87: 13$ \\
C & Et & 1.0 & $62: 38$ \\
D & Et & 0.1 & $74: 26$ \\
E & i-Pr & 0.1 & $65: 35$ \\
F & i-Bu & 0.1 & $70: 30$ \\
G & t-Bu & 0.1 & $82: 18$ \\
\hline
\end{tabular}

Table 1, entry C and D). These values do not correspond to the spin-characteristic diasteroselectivities but are close to them, indicating that high endo-selectivity is characteristic to triplet photocycloadditions, whereas in case of the singlet excited carbonyl compounds, the diasteroselectivity approaches a $50: 50$ ratio.

The diastereomeric ratios for four different aliphatic aldehydes 1c-f showed only marginal substituent effects, the tert.-butyl derivative (i.e., pivaldehyde 1f) gave the highest endo-selectivities, contraintuitive from a ground-state point of view, but in agreement with the ISC-geometry model as shown in Figure 1.

The diastereomeric ratios of the oxetanephotoadducts were determined by ${ }^{1} \mathrm{H}$-NMR spectroscopy and the product structures were identified from ${ }^{1} \mathrm{H}$-NMR and ${ }^{13} \mathrm{C}$-NMR spectral analyses. The relative configurations of the diastereoisomers exo-3c and endo-3c were unambiguously determined from NOE difference measurement. In the exo-3c isomer, irradiation of the methyl protons at $1.51 \mathrm{ppm}$ led to nuclear Overhauser enhancements of both methyl protons signal at $1.22 \mathrm{ppm}$ and the methine proton signal, $\mathrm{H}-7$ at $4.16 \mathrm{ppm}$. Likewise, in endo-3c, irradiation of a methyl proton signal at $1.45 \mathrm{ppm}$ led to nuclear Overhauser enhancement of the methyl signal at $1.14 \mathrm{ppm}$ (see Figure 2).

\section{MECHANISTIC ANALYSIS}

The idealized structures for the triplet 1,4-biradical conformers prior to intersystem crossing (ISC) are shown in Figure 3. 

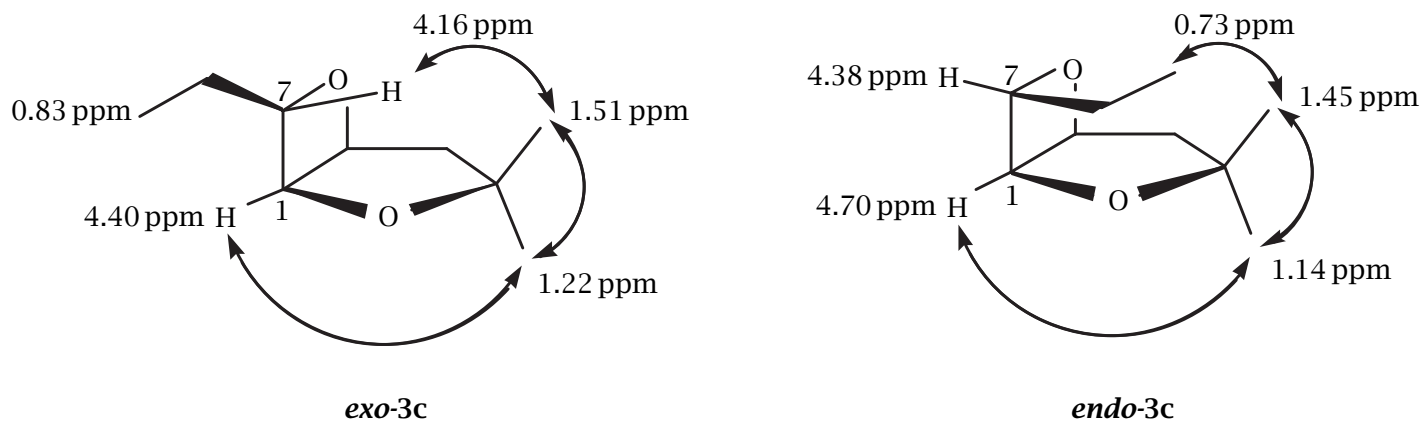

Figure 2. NOE interactions in bicyclic oxetanes exo-3c and endo-3c.

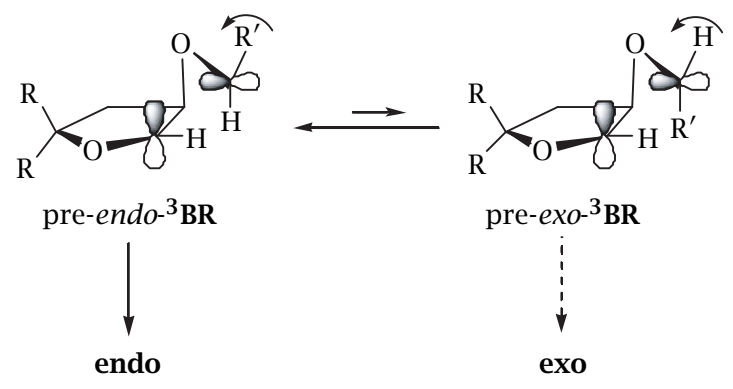

Figure 3. Triplet 1,4-biradical precursors to diastereoisomeric oxetanes.

Obviously, the most decisive interactions determining the product stereochemistry are between the $\alpha$ hydrogen and the substituent $\mathrm{R}^{\prime}$ at $\mathrm{C} 4$ of the oxatetramethylene biradical. An increase in steric bulk of $\mathrm{R}^{\prime}$ is expected to lead also to an increase in the (thermodynamically less favoured) endo-diastereoisomer. This was verified for the tert.-butyl substituted oxetanes $\mathbf{3 f}$ in comparison with the ethylated derivatives 3c. Likewise, in the pure triplet photocycloadditions of $\mathbf{2 a}$ and $\mathbf{2 b}$, the insertion of an ortho-methyl group led to an increase in endo-selectivity, i.e., an increase in population of the pre-endo- ${ }^{3} \mathbf{B R}$. On the other hand, two additional methyl groups at $\mathrm{C}-2$ of the cycloalkene is not recognized in the critical biradical conformers and thus, does not alter the diastereoisomeric ratios.

\section{CONCLUSION}

The simple (non-induced) diastereoselectivity of the photocycloaddition of triplet excited aldehydes to 2,2dimethyl-2,3-dihydrofuran (1) is high and contrathermodynamic (endo) due to the spin-orbit coupling controlled ISC geometries of the intermediary triplet 1,4biradicals leading to ground state products (oxetanes 3). By variation of the substrate concentration (spin mapping) the reactivity of the corresponding singlet ex- cited carbonyls was estimated and shown to be considerably lower.

\section{REFERENCES}

[1] A. G. Griesbeck and S. Bondock, Handbook of Photochemistry and Photobiology (W. M. Horspool and F. Lenci, eds.), CRC Press, Boca Raton, 2003, Chapter 60, pp. 1-21.

[2] A. G. Griesbeck and M. Fiege, Mol. Supramol. Photochem. (V. Ramamurthy and K. S. Schanze, eds.), 6, 2000, p. 33; J. A. Porco and S. L. Schreiber, Comprehensive Organic Synthesis (B. M. Trost and I. Fleming, eds.), Pergamon, New York, 5, 1991, p. 151; T. Bach, Synthesis (1998), 683.

[3] A. G. Griesbeck, M. Fiege, S. Bondock, and M. S. Gudipati, Org. Lett. 2 (2000), 3623.

[4] A. G. Griesbeck and S. Bondock, J. Am. Chem. Soc. 123 (2001), 6191.

[5] A. G. Griesbeck and S. Bondock, Photochem. Photobiol. Sci. 1 (2002), 81.

[6] A. G. Griesbeck, S. Bondock, and M. S. Gudipati, Angew. Chem., Int. Ed. 40 (2001), 4684.

[7] A. G. Griesbeck, S. Bondock, and P. Cygon, J. Am. Chem. Soc. 126 (2003), 9016.

[8] I. J. Palmer, I. N. Ragazos, F. Bernardi, M. Olivucci, and M. A. Robb, J. Am. Chem. Soc. 116 (1994), 2121.

[9] A. G. Griesbeck, H. Mauder, K. Peters, E.-M. Peters, and H. G. von Schnering, Chem. Ber. 124 (1991), 407.

[10] S. Buhr, A. G. Griesbeck, J. Lex, J. Mattay, and J. Schröer, Tetrahedron Lett. 37 (1996), 1195.

[11] A. G. Griesbeck and S. Stadtmüller, J. Am. Chem. Soc. 113 (1991), 6923.

[12] A. G. Griesbeck, H. Mauder, and S. Stadtmüller, Acc. Chem. Res. 27 (1994), 70.

[13] A. Kutateladze, J. Am. Chem. Soc. 123 (2001), 9279.

[14] S.-K. Park, S.-J. Lee, K. Baek, and C.-M. Yu, Bull. Korean Chem. Soc. 19 (1998), 35.

[15] J. Huet, Bull. Soc. Chim. Fr. 26 (1964), 447.

[16] A. G. Griesbeck and S. Stadtmüller, Chem. Ber. 123 (1990), 357. 



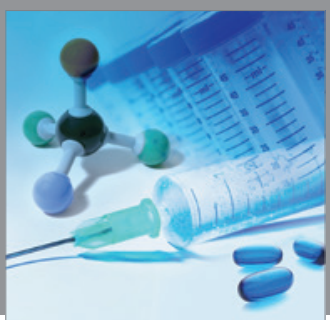

International Journal of

Medicinal Chemistry

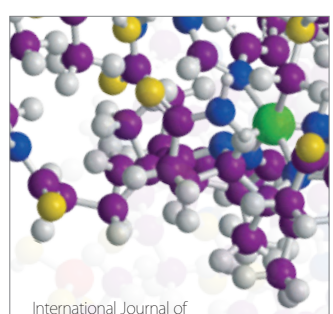

Carbohydrate Chemistry

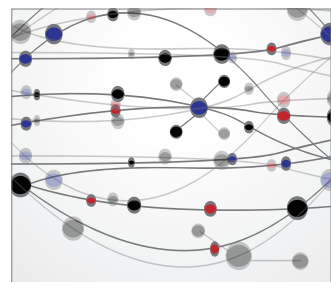

The Scientific World Journal
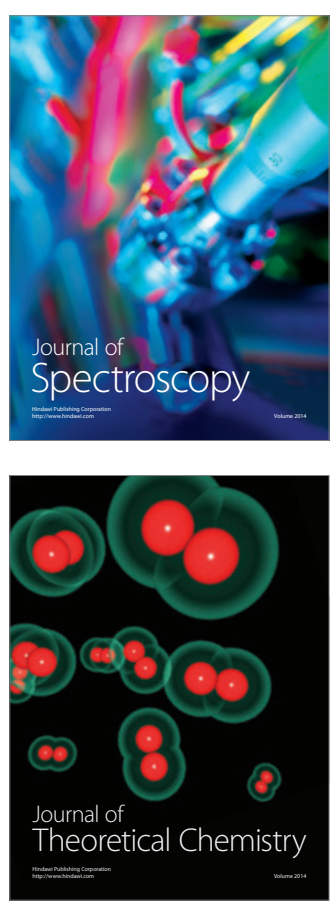
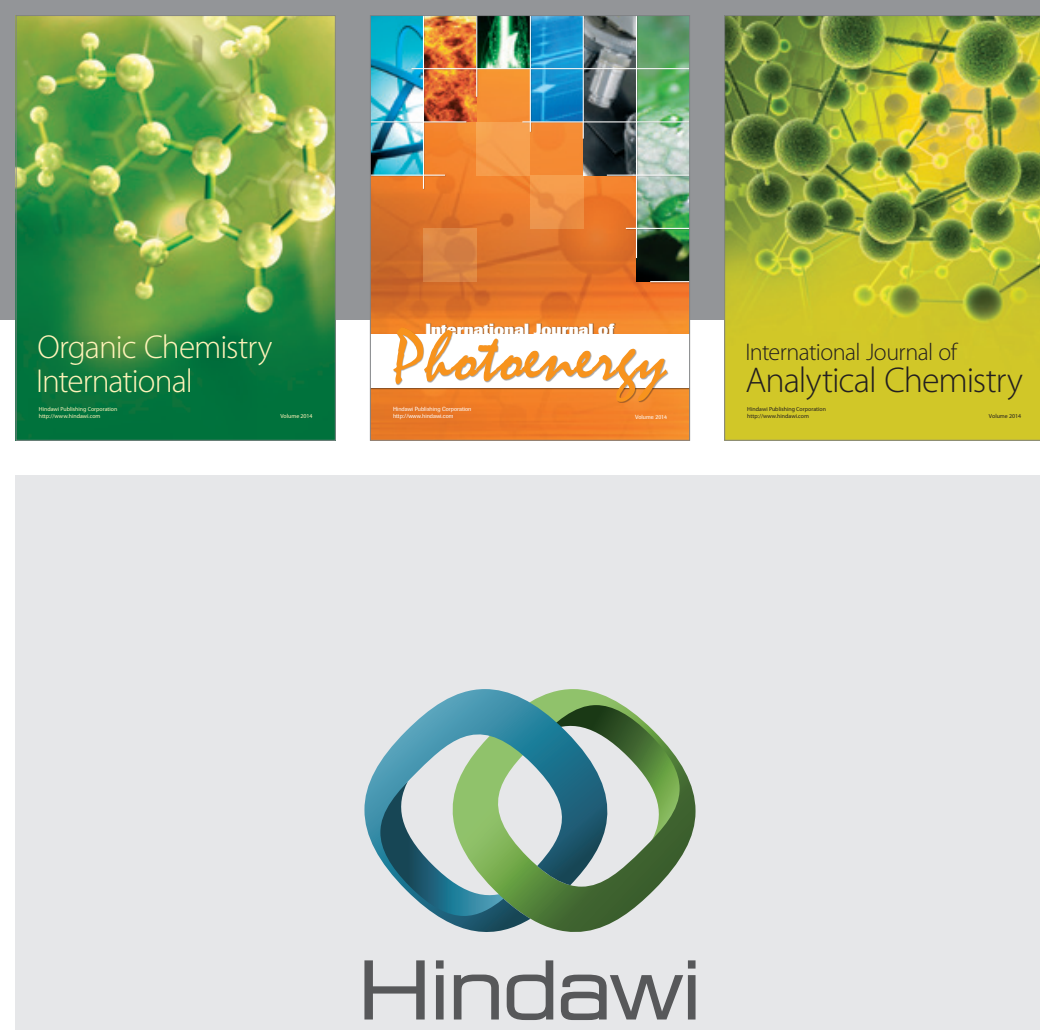

Submit your manuscripts at

http://www.hindawi.com
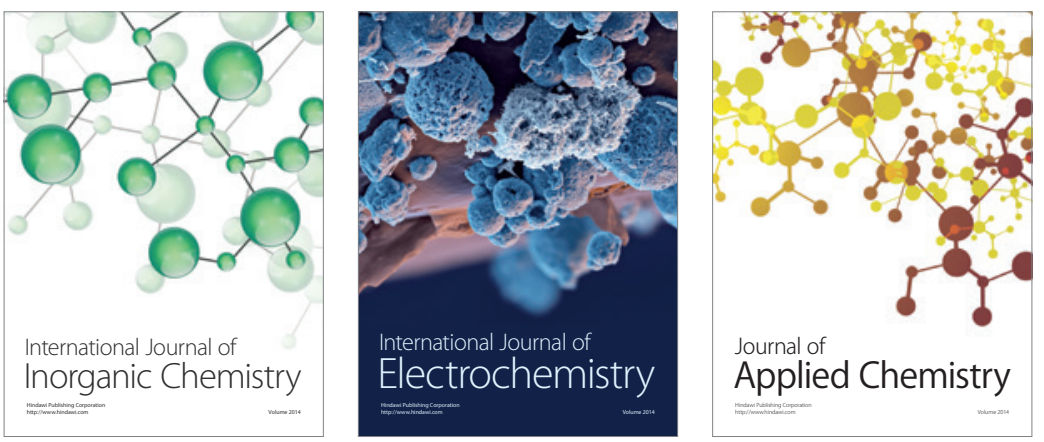

Journal of

Applied Chemistry
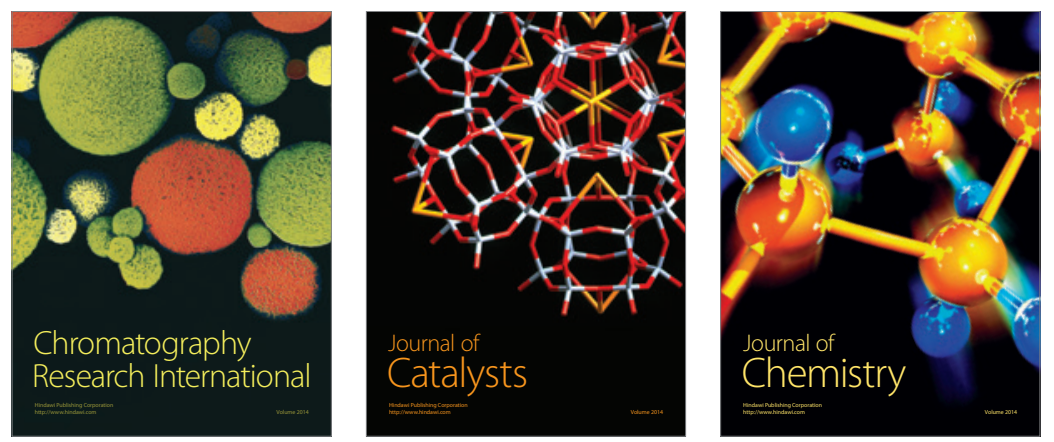
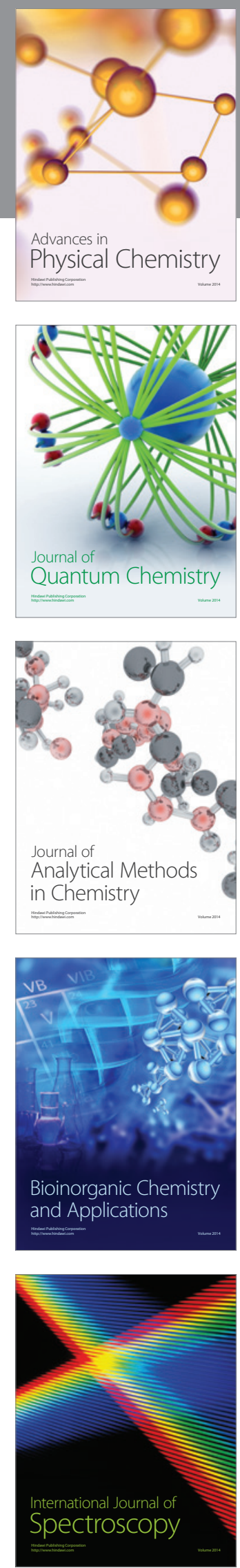